\title{
Identification of Cellular Processes That Are Rapidly Modulated in Response to Tracheal Occlusion Within Mice Lungs
}

\author{
TOMMY SEABORN, JONNY ST-AMAND, MARC CLOUTIER, MICHEL G. TREMBLAY, FRANCE MALTAIS, \\ STÉPHANIE DINEL, VÉRONIQUE MOULIN, PAUL A. KHAN, AND BRUNO PIEDBOEUF
}

\begin{abstract}
Pediatric Research Unit [T.S., M.C., F.M., P.A.K., B.P.], Functional Genomics Laboratory [J.S.-A., M.G.T., S.D.], Laval University, Ste-Foy, Québec, G1V 4G2, Canada; Laboratoire des grands brulés/LOEX [V.M.], Laval University, Ste-Foy, Québec, G1S 4L8, Canada
\end{abstract}

\begin{abstract}
Lung development progresses through a process reliant on mechanical cell stretch. However, this process is not well defined at the molecular level. Our goal was to globally analyze the transcriptome of fetal mouse lungs following short periods of tracheal occlusion (TO) to identify cellular processes that are rapidly modulated in response to intraluminal stretch increase. Serial analysis of gene expression (SAGE) was used to examine the global transcriptomic response of mouse prealveolar stage lungs to in vivo TO at 1 and $3 \mathrm{~h}$. SAGE results were extended by histo- and immunochemical examination. Based on the 97 TO-modulated transcripts identified, our results further point out that continuous stretch in developing lungs leads directly to rapid and highly specific phenotypic modifications in a significant proportion of pulmonary cells. We conclude that intraluminal stretch increase during prealveolar stage of lung development induces a critical transition of pulmonary cells phenotype in which there is an increase in $\alpha$-smooth muscle actin ( $\alpha$-SMA)-containing cells along with a relative decrease in lipidcontaining cells. (Pediatr Res 63: 124-130, 2008)
\end{abstract}

$\mathrm{A}$ $t$ the end of gestation, fetal lung volume is under the influence of an important and continuous distention that is secondary to the continuous intraluminal liquid secretion by the fetal lung epithelium (1). Within developing lungs, this liquid sequestration occurs by the active larynx closure during the apnea (1), which accounts for the major portion of late fetal life (2). Abnormal loss of lung liquid is detrimental to lung development (3). Indeed, fetal cervical cord transection, which results in a lack of coordination between larynx opening and diaphragmatic movements, thus allowing excessive loss of lung liquid during fetal breathing movements, leads to lung hypoplasia (4). Similarly, loss of larynx tonus (5) and bypass of the larynx by experimental tracheotomy (6) during fetal life are both associated with excessive lung liquid loss and severe lung hypoplasia. In contrast, stimulation of lung growth and development can be achieved by using tracheal

Received April 9, 2007; accepted September 11, 2007.

Correspondence: Bruno Piedboeuf, M.D., CHUL Research Center, Pediatric Research Unit, 2705 Laurier Blvd., RC-9800, Québec, Québec, G1V 4G2, Canada; e-mail: Bruno.Piedboeuf@crchul.ulaval.ca

This work was supported by the Canadian Institutes of Health Research, le Fonds de Recherche en Santé du Québec, the Jeanne and Jean-Louis Lévesque Chair in Perinatalogy, and the Foundation for Research into Children's Diseases. obstruction techniques at an appropriate developmental stage (7). By sequestering intraluminal liquid, fetal tracheal occlusion (TO) amplifies continuous stretch. TO represents a unique in vivo methodology that facilitates examination of the natural three-dimensional mechanical forces effect on lung development.

Although the relationship between the degree of fetal lung expansion and lung development is well established, the mechanisms involved are not well understood. We hypothesize that an increase in fetal lung stretch causes a stimulus that rapidly mediates gene expression before any change in fetal lung structure is observed. To investigate the mechanisms underlying stretch-induced lung maturation, we previously created an in vivo murine model of TO that demonstrated a significant structural acceleration of prealveolar stage lung growth and development in a 24-h period (8). With this model, we recently demonstrated that TO induces an immediate cellular response (9). Indeed, when compared with sham-TO and unoperated-on controls, TO induced an increase in proliferating cell nuclear antigen (PCNA) protein expression in differentiated epithelial airways within the first hour after surgery. Here, our aim was to globally analyze the transcriptome of fetal mouse lungs after precisely defined short periods of TO to identify cellular processes that are rapidly modulated in response to intraluminal stretch increase.

To identify the full module of mechanically transduced genes, we compared transcriptomes of TO and sham-TO fetal lungs at 1 and $3 \mathrm{~h}$ post-surgery using serial analysis of gene expression (SAGE). Transcripts differentially expressed between correspondingly timed sham-TO and TO fetal lungs were categorized by gene function. The major changes observed concern cytoskeleton and metabolism related transcripts. Because pulmonary interstitial fibroblasts are known to present a switch from lipogenic to myogenic phenotypes after birth (10), SAGE results were extended by histo- and immunochemical analysis of both cellular lipids and contractile interstitial cells.

\footnotetext{
Abbreviations: $\boldsymbol{\alpha}$-SMA, $\alpha$-smooth muscle actin; DAB, 3,3 $3^{1}$-diaminobenzidine; ORO, oil red O; SAGE, serial analysis of gene expression; TO, tracheal occlusion
} 


\section{MATERIALS AND METHODS}

Surgical procedure and tissue harvesting. The Committee for Animal Protection of Laval University approved the surgeries. TO was performed on CD-1 mice at $16.5 \mathrm{~d}$ of gestation (late canalicular stage of lung development) as described previously (8). The fetal trachea was occluded using a surgical ligation clip. The fetus was returned to the uterus where gestation continued. Control sham-TO fetuses underwent exactly the same surgery save actual TO. Dams were killed 1 or $3 \mathrm{~h}$ after surgery and operated fetuses were delivered by cesarean. The success of TO over such a short time frame (i.e. before gross morphologic changes appear) was previously confirmed by the observation of a significant increase in luminal space fraction by only $1 \mathrm{~h}$ of TO when lungs were fixed with a surgical clip left in place (TO cases) or added before the harvesting (sham-TO cases) (9). In addition, fetal neck was subjected to a careful examination, as described previously (8). Fetal lungs were harvested, snap-frozen in liquid nitrogen, and stored at $-80^{\circ} \mathrm{C}$. Lungs used for SAGE, neutral lipid staining, and $\alpha$-smooth muscle actin ( $\alpha$-SMA) immunofluorescence and immunohistochemistry came from different animals because the tissue-fixation process differed among experiments.

$\boldsymbol{S A G E}$. SAGE is a method that gives an overview of a sample's gene activity by capturing mRNAs and counting and identifying them. The four SAGE libraries were separately generated as previously described (11) from pooled fetal lungs (10 in each experimental TO and sham-TO group at 1 and 3 h). Resulting SAGE tags 15-bp long were matched in GenBank and UniGene databases to identify corresponding transcripts. Reliable tag-to-gene matching was attributed to tags corresponding with a polyadenylated sequence and whose last $3^{\prime}$ NlaIII site was on the positive sequence strand. When a tag did not meet these criteria for at least one expressed sequence tag with a known poly- $\mathrm{A}^{+}$tail in the UniGene cluster, it was not considered further. To compare tag differential expression levels among libraries, data were normalized to 50,000 tags per library. To detect the transcripts upregulated or down-regulated in response to TO with more than a twofold change in tag levels, a comparative count display test was used (11). Differences among control and experimental libraries were considered statistically significant at $p<0.05$. Tag-to-gene matches have been categorized by gene function based on information reported by Gene Ontology and TIGR Web sites.

Identification of lipid-containing cells. Lipogenic cell phenotype was specifically assessed using oil red O (ORO) staining (12). Lungs were embedded in OCT medium and frozen at $-80^{\circ} \mathrm{C}$, and sections were cut. Sections were fixed in formalin, rinsed in water, soaked in propylene glycol, and immersed in ORO (FluKa Chemica, Oakville, Canada) to color neutral lipids (mainly triglycerides). Sections were rinsed in propylene glycol and water and counterstained with hematoxylin. Nuclei were stained with Hoechst. Results were expressed as the ratio of the pixel area of ORO staining, which autofluoresces at 570-580 nm, over the pixel area of the nuclei (13) to avoid potential bias resulting from difference in luminal space over tissue fraction between sham-TO and TO groups. The numbers of fetal lungs analyzed were 11 for 1 -h TO, 10 for 1-h sham-TO, 10 for 3-h TO, 11 for 3-h sham-TO, 11 for 24-h TO, and eight for 24-h sham-TO.

Identification of $\alpha$-SMA-containing cells. Indirect immunofluorescence was performed on methanol-fixed cryosections as described previously (14). Mouse monoclonal anti- $\alpha$-SMA antibody (clone 1A4, DakoCytomation, Glostrup, Denmark) was used. Secondary antibody was dichlorotriazinylaminofluorescein-conjugated goat anti-mouse IgG-IgM (Chemicon, Temecula, CA). Nuclei were stained with Hoechst. Results were expressed as the ratio of the pixel area of $\alpha$-SMA staining (520-530 nm) over the pixel area of the nuclei (15). Digital images were captured using the red channel to optimize contrast. The numbers of fetal lungs analyzed were 10 for 1-h TO and sham-TO, nine for 3-h TO, and 10 for 3-h sham-TO. We also performed 3,3(1)-diaminobenzidine (DAB)-based immunohistochemistry to allow visualization of the tissue context of $\alpha$-SMA protein expression pattern. Formalinfixed and paraffin-embedded lung sections were deparaffinized in xylene and rehydrated in a decreasing ethanol gradient. Endogenous peroxidase activity was blocked with $0.6 \%$ hydrogen peroxide $/ 100 \%$ methanol. After blocking (Tris-buffered saline, $0.5 \%$ bovine serum albumin, $1.5 \%$ normal goat serum), sections were incubated with the anti- $\alpha$-SMA antibody. The sections were then incubated with biotinylated secondary antibody and revealed with a Vectastain ABC Kit (Vector Laboratories, Burlingame, CA). After incubation in a streptavidin-peroxidase solution, the slides were developed with DAB. Counterstaining was performed with hematoxylin. The numbers of fetal lungs observed were six for 1-h TO, five for 1-h sham-TO, six for 3-h TO, and seven for 3-h sham-TO. For each immunostaining, two control sections were identically treated except that blocking serum replaced primary antibody in one and secondary antibody in the other. Negligible background was observed for all controls.

Microscopic analyses. Lung section randomization and analyses were conducted according to guidelines of Bolender et al. (16) to minimize observer bias. On average, 10 randomized fields per fetus were analyzed from a minimum of eight lungs in each of the experimental and control groups that was used for quantification. Data regarding ORO fluorescence and immunofluorescence of $\alpha$-SMA protein were analyzed by analysis of variance and Scheffé post hoc tests (SAS software) performed on the averaged data from each animal. Significance was deemed at $p<0.05$.

\section{RESULTS}

Approximately 39,000-50,000 tags from each of the four SAGE libraries were sequenced for a total of 186,189 tags (Table 1). The percentage of tag species that correspond with known mRNA sequence(s) in GenBank and UniGene databases was similar among the four libraries (range, 32\%-39\%). The number of tags present at a level of expression $>0.5 \%$ was also consistent among the four libraries (range, six to 12) as well as the number of those showing an expression level $>0.1 \%$ (range, $63-77$ ).

As our interests concern early transcriptional response to intraluminal stretch increase during late lung development, we compared TO libraries with their corresponding sham-TO libraries. Overall, a total of 97 unique tags were modulated significantly at 1 and/or $3 \mathrm{~h}$ post-TO $(p<0.05)$. We observed 66 tags modulated after only $1 \mathrm{~h}$ of TO: 36 were up-regulated and 30 down-regulated. In addition, we observed 60 tags modulated by $3 \mathrm{~h}$ of TO: 27 were up-regulated and 33 down-regulated. To delineate the cellular processes that are rapidly modulated by TO, differentially expressed tags have been organized by functional families (Fig. 1). Based on this classification, tags modulated by 1 and $3 \mathrm{~h}$ of TO correspond to transcripts related to six major functional families: cell structure and motility, metabolism, cell signaling and communication, cell cycle, RNA and protein synthesis, and cell and organism defense. Other TO-modulated tags correspond to transcripts for which the biologic function has not been yet characterized.

Tags functionally corresponding with cell structure and motility (cytoskeleton in Figure 1) represent an important

Table 1. Characterization of SAGE libraries obtained from fetal mouse lungs following 1 and 3 h of in utero TO or sham-TO surgery

\begin{tabular}{|c|c|c|c|c|c|c|c|c|}
\hline \multirow[b]{2}{*}{ Library } & \multirow{2}{*}{$\begin{array}{c}\text { No. of } \\
\text { sequenced tags }\end{array}$} & \multirow{2}{*}{$\begin{array}{c}\text { No. of } \\
\text { distinct tags }\end{array}$} & \multicolumn{2}{|c|}{ Genes identified } & \multicolumn{2}{|c|}{$\begin{array}{c}\text { Tags with an } \\
\text { expression level }>0.5 \%\end{array}$} & \multicolumn{2}{|c|}{$\begin{array}{c}\text { Tags with an } \\
\text { expression level }>0.1 \%\end{array}$} \\
\hline & & & No. & $\% *$ & No. & $\% *$ & No. & $\% *$ \\
\hline 1-h TO & 39,119 & 18,259 & 6020 & 33 & 7 & 0.04 & 65 & 0.4 \\
\hline 1-h sham-TO & 50,411 & 24,694 & 7984 & 32 & 12 & 0.05 & 63 & 0.3 \\
\hline 3-h TO & 47,594 & 20,698 & 6527 & 32 & 12 & 0.06 & 77 & 0.4 \\
\hline 3-h sham-TO & 49,065 & 24,013 & 9478 & 39 & 6 & 0.02 & 66 & 0.3 \\
\hline
\end{tabular}

\footnotetext{
* The percentage is the number $\times 100$ over the number of distinct tags.
} 
Up-Regulated
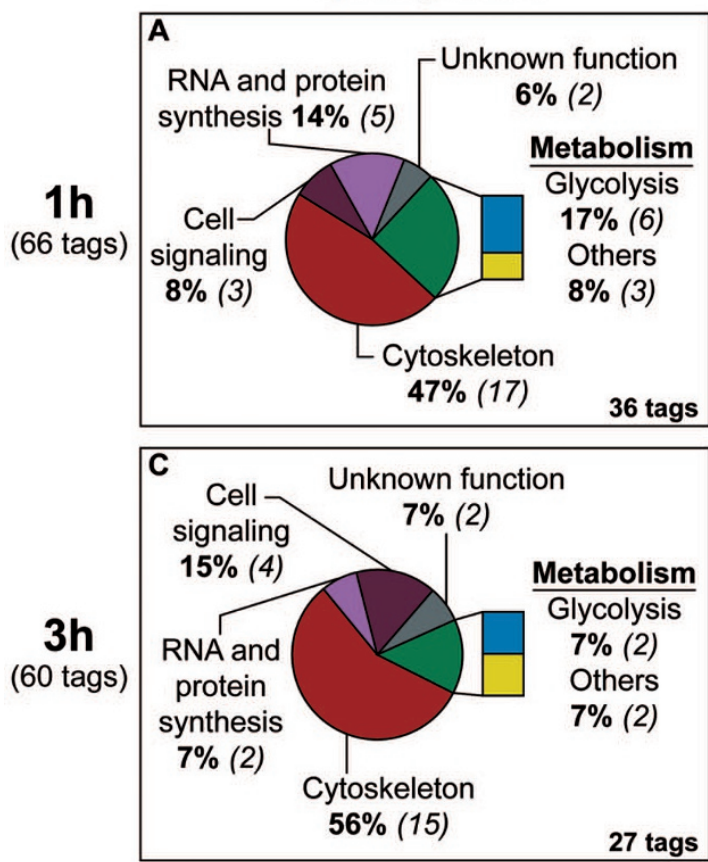

Down-Regulated
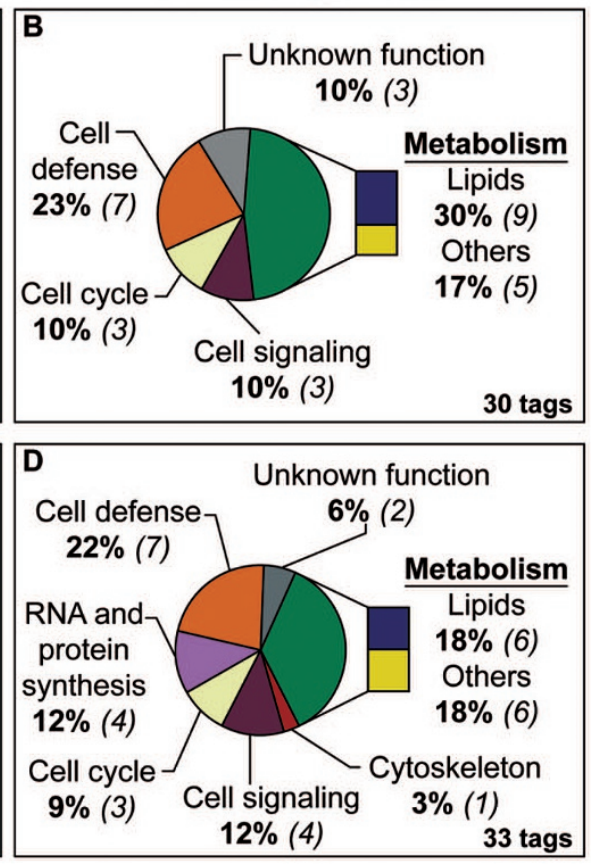

Figure 1. Distribution of TO-modulated transcripts into functional families. Distribution of functional families of transcripts corresponding with unique SAGE tags modulated by TO compared with sham-TO $(p<0.05)$. The number of tags modulated in each functional family appears in parentheses. proportion of tags up-regulated by TO: $47 \%$ of tags upregulated by $1 \mathrm{~h}$ of TO (17 tags) and 56\% of tags up-regulated by $3 \mathrm{~h}$ of TO (15 tags). Among the 17 tags up-regulated by $1 \mathrm{~h}$ of TO, 14 remained higher in the TO library than in the sham-TO library at $3 \mathrm{~h}$. Interestingly, eight of these transcripts were fast isoforms of muscle-related proteins.

Tags corresponding with metabolism related transcripts account for $25 \%$ and $14 \%$ of the tags up-regulated by TO at 1 and $3 \mathrm{~h}$, respectively and for $47 \%$ and $36 \%$ of the tags down-regulated by TO at 1 and $3 \mathrm{~h}$, respectively. The major metabolic response observed with TO concerns up-regulation of glycolysis and down-regulation of lipid metabolism. Indeed, six tags corresponding with glycolysis-related transcripts were up-regulated by $1 \mathrm{~h}$ of TO. In contrast, lipid metabolism-related transcripts were essentially downregulated by TO: nine tags at $1 \mathrm{~h}$ and six tags at $3 \mathrm{~h}$. Four tags were down-regulated by 1 and $3 \mathrm{~h}$ of TO and five tags were down-regulated by $1 \mathrm{~h}$ of TO only. Tags corresponding to genes related to metabolic processes other than glycolysis and lipids were also up- and down-regulated by both 1 and $3 \mathrm{~h}$ of TO. They principally represent energy metabolism, but also amino acid and carbohydrate metabolic processes.

As mentioned previously, tags corresponding with proteins related to other biologic processes were also rapidly modulated by TO. For example, some tags corresponding with genes related to RNA and protein synthesis as well as to cell signaling were up-regulated by TO at both 1- and 3-h time points. Conversely, tags corresponding with genes encoding for cell defense-related proteins, cell cycle and cell signaling were down-regulated by TO at both 1- and 3-h time points. We also observed four tags corresponding with genes related to RNA and protein synthesis and another one corresponding with cytoskeletal protein that were down-regulated only at $1 \mathrm{~h}$ of TO. Finally, we observed few tags up- and down-regulated by both 1 and $3 \mathrm{~h}$ of TO for which the corresponding protein function is unknown.

ORO coloration shows a temporal increase in neutral lipid (primarily triglyceride) content from 1 to $24 \mathrm{~h}$ in both sham-TO and TO lungs (Fig. 2). The staining is restricted to mesenchyme and is virtually absent in epithelium. At 1 and $3 \mathrm{~h}$, no significant difference was observed among TO and sham-TO groups (Fig. 2G). However, the neutral lipid content of the TO group after $24 \mathrm{~h}$ is lower than that of the sham-TO group $(p<0.05)$. Myogenic phenotype was visualized by $\alpha$-SMA bright-field immunohistochemistry (Fig. 3A-D) and quantified by using immunofluorescence (Fig. $3 E-H$ ). $\alpha$-SMA is considered a marker for the initial differentiation of smooth muscle cells and is also detected in alveolar myofibroblasts and microvascular pericytes (17). A significant increase in density of cells expressing $\alpha$-SMA occurs in TO cases when compared with controls (Fig. 3I). This increase is observed $1 \mathrm{~h}$ after surgery and is sustained at $3 \mathrm{~h}(p<0.05)$. However, Western blot analysis at 1 and $3 \mathrm{~h}$ does not show significant difference in $\alpha$-SMA protein total amount between sham-TO and TO lungs (data not shown). In sham-TO cases, $\alpha$-SMA staining is virtually restricted to the mesenchyme surrounding developing airway epithelium (Fig. $3 B$ and $D$ ) while, in TO group, it appears also within the mesenchyme surrounding acini in lung periphery (Fig. $3 A$ and $C$ ).

\section{DISCUSSION}

SAGE is a well-established technology allowing rapid and efficient identification as well as quantitative assessment of genes expressed during developmental, physiologic, or pathologic conditions $(11,18)$. Here, SAGE was used to obtain a prospective overview of cellular processes that are rapidly 

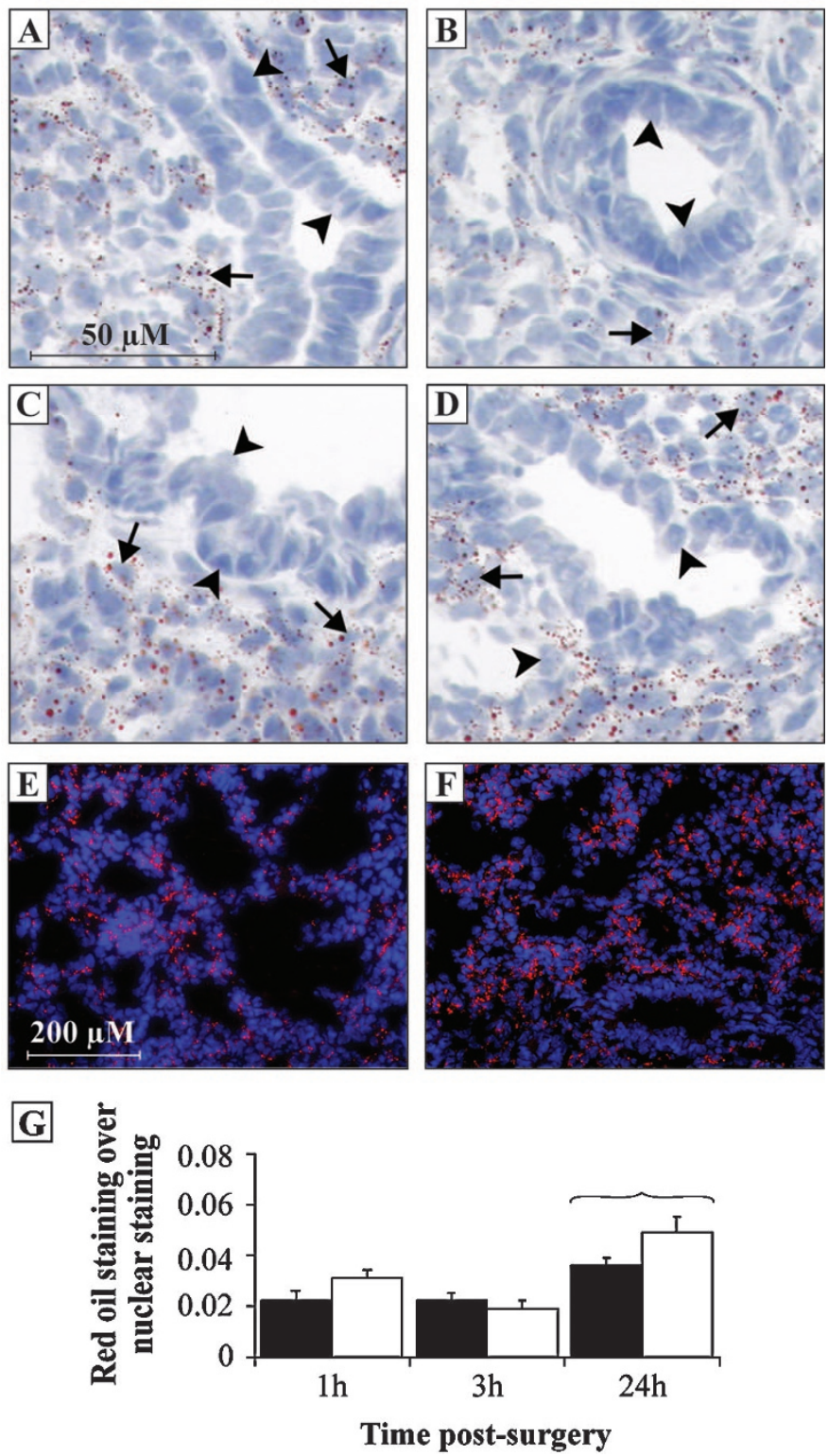

Figure 2. Assessment of lipid-containing cells within the lung in response to TO. ORO triglyceride staining in mouse lungs after TO (left column) and sham-TO (right column). Examples after $1 \mathrm{~h}(A, B)$ and $24 \mathrm{~h}(C-F)$ observed in both bright-field $(A-D)$ and fluorescence $(E, F)$. Nuclei are stained blue with hematoxylin (bright field) and Hoechst (fluorescence). Triglyceride staining appears as dark spots in bright field and red in fluorescence. $(G)$ Ratio of the pixel area of ORO staining over the pixel area of nuclei (mean \pm SEM). Brace indicates significant difference $(p<0.05)$ between TO and sham-TO. Arrows, mesenchymal cells; arrowheads, epithelial cells; solid columns, TO, open columns, sham-TO.

modulated within the lung by pulmonary intraluminal stretch increase resulting from TO.

The proportion of tags matching with sequences in GenBank and UniGene databases reported here is consistent with other SAGE studies $(11,19,20)$. Moreover, the reproducibility of SAGE methodology has been established previously by us (21) and by others (22). Furthermore, consistency of the differentially expressed tags identification in the four independent libraries corroborates the validity and reproducibility of SAGE results. Finally, histo- and immunochemistry demon-
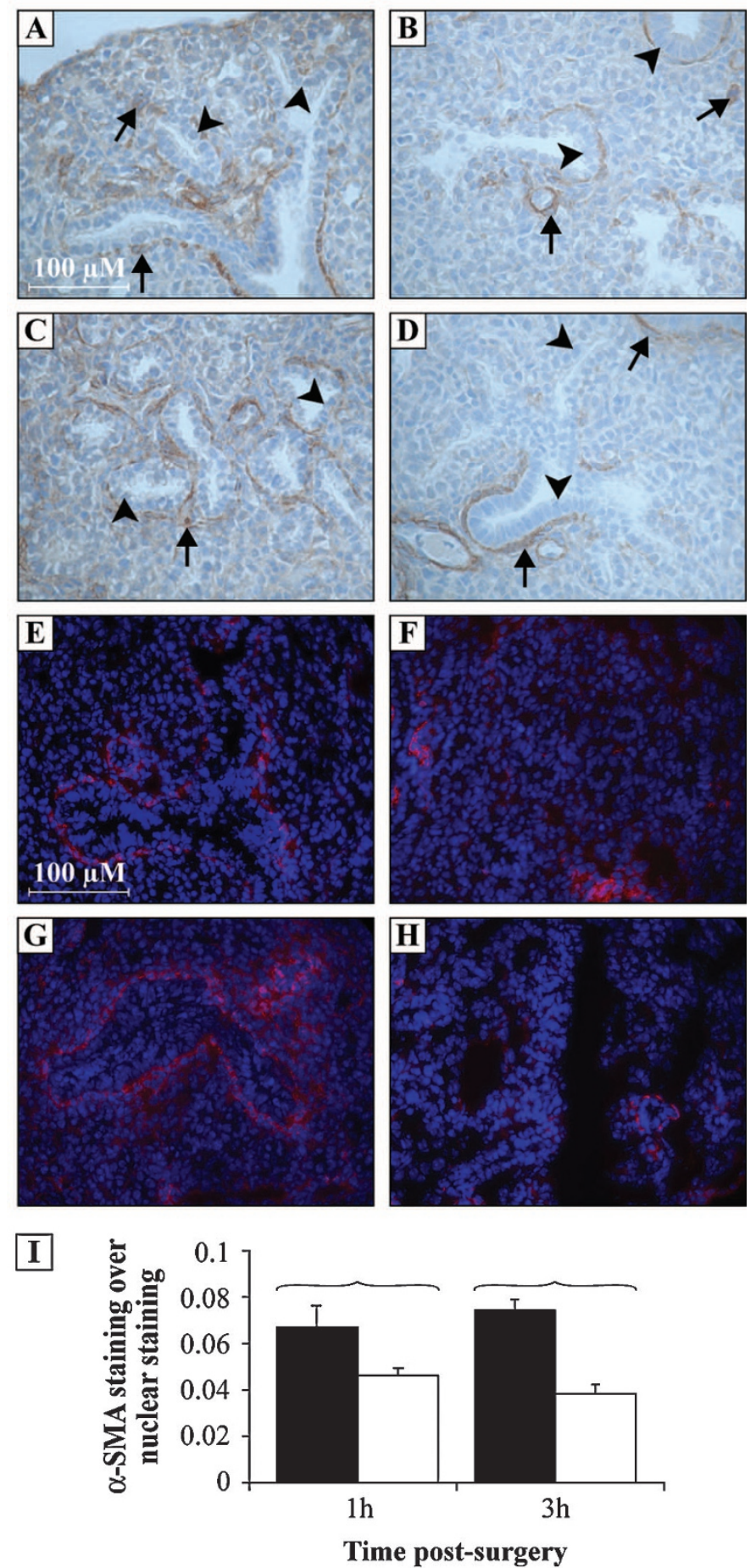

Figure 3. Assessment of $\alpha$-SMA-containing cells within the lung in response to TO. $\alpha$-SMA staining in mouse lungs after TO (left column) and sham-TO (right column). Examples after $1 \mathrm{~h}(A, B)$ and $3 \mathrm{~h}(C, D)$ visualized by DAB-based immunohistochemistry. Examples after $1 \mathrm{~h}(E, F)$ and $3 \mathrm{~h}(G, H)$ by using immunofluorescence in which nuclei are stained blue with Hoechst and $\alpha$-SMA staining appears as red. (I) Ratio of the pixel area of $\alpha$-SMA over the pixel area of nuclei (mean \pm SEM). Braces indicate significant difference $(p<0.05)$ between TO and sham-TO. Arrows, mesenchymal cells; arrowheads, epithelial cells; solid columns, TO; open columns: sham-TO.

strate that changes observed in lipid- and $\alpha$-SMA-containing cells are consistent with SAGE results.

Our results demonstrate that transcriptional response to TO is triggered within $1 \mathrm{~h}$ and sustained to a comparable intensity 
for at least $3 \mathrm{~h}$. This agrees with in vitro studies that show rapid gene regulation and protein activation after stretch of fetal lung cells in culture (23). The number of identified transcripts modulated in response to TO underline a rapid and important transcriptional response in pulmonary cells to intraluminal stretch increase. Classification of these TOmodulated tags according to their specific corresponding genes function suggests principally that TO induces 1) a modification of the cytoskeleton with a noteworthy increase in cellular levels of fast muscle isoforms and 2) a metabolic switch from cellular lipid synthesis and accumulation to glycolysis.

Tags corresponding with cell structure and motility genes represent an important portion of all tags up-regulated by TO. This suggests that reorganization of cytoskeleton and/or cell motility take place in response to mechanotransduction within the lung. At both time points examined, a substantial portion of differentially expressed up-regulated tags matches cytoskeletal transcripts. Almost exclusively, these tags are associated with contractile apparatus and, in large part, correspond with actin-, myosin-, and troponin-associated genes. Thus, it appears that mechanotransduction induced by TO supports upregulation of muscle phenotype.

Examination of TO-regulated tags corresponding with metabolism-related transcripts suggests that a rapid metabolic response occurs because of TO-induced mechanotransduction. Specifically, the six tags corresponding with transcripts related to glycolysis, which are up-regulated by $1 \mathrm{~h}$ of TO (aldolase 1, enolase 3, glyceraldehyde-3-phosphate dehydrogenase, phosphofructokinase, phosphoglycerate mutase 2, and pyruvate kinase) account for six of the 10 enzymes essential for glucose to pyruvate metabolism (Table 2). This suggests that TO stimulates the glycolytic pathway in pulmonary cells. This is reinforced by the TO increase in abundance of fast isoforms of muscle-related proteins, which are characteristic of glycolytic tissue (24). TO-down-regulated transcripts related to lipid metabolism correspond with enzymes or proteins in- volved in synthesis, storage, and trafficking of lipids. This suggests that TO is associated with a down-regulation of both lipid synthesis and storage in pulmonary cells as well as with lipid trafficking modulation. These data propose that TO induces a metabolic switch from cellular lipid synthesis and accumulation to glycolysis within the lung. This is supported by the decrease in lipid-containing cells and the increase in $\alpha$-SMA-containing cells observed by immuno- and histochemistry.

Because glycolysis is an anaerobic fermentation process and energy production by lipid metabolism is an oxidative process, the metabolic switch that we report could result from TO-induced tissue hypoxia. Indeed, it has been reported that liquid expansion of fetal lung results in increased vascular resistance and thus reduced pulmonary blood flow (25). However, it is unlikely that the increase in the small luminal space fraction observed after $1 \mathrm{~h}$ of TO $(4.0 \pm 0.4$ versus $2.7 \pm 0.3$ for the sham-TO group; $p<0.01)$ causes significant compression of the blood vessel (9). Moreover, lungs subjected to short-term TO (Figs. 2 and 3) or to longer term TO (24 and $36 \mathrm{~h}$ ) (8) do not show any morphologic deleterious effect when compared with sham-TO lungs. In addition, hypoxia-inducible factors (e.g. HIF- $1 \alpha$, HIF- $1 \beta$, HIF- $2 \alpha$, HIF-3 $\alpha$ ) were neither up- nor down-regulated by TO in our study. Taken together, these observations argue against a hypoxic effect of short-term TO within the fetal lung.

Expression of tags related to various other processes is modulated by TO. These tags concern cell signaling and cell communication, cell cycle, RNA and protein synthesis, and cell and organism defense. This confirms that a rapid, complex, and important pulmonary response occurs after mechanotransduction during late lung development. Transcripts encoding for proteins related to cell and organism defense account for the most important part of these other processes modulated by TO. It is hard to assert about the role of cell defense-related proteins in the developmental lung process. Interestingly, these tags showed a lower expression level in

Table 2. Examples of transcripts significantly up-regulated in response to TO as revealed by SAGE

\begin{tabular}{|c|c|c|c|c|c|c|}
\hline \multirow[b]{3}{*}{ Transcript (accession no.) } & \multicolumn{3}{|c|}{$1 \mathrm{~h}$} & \multicolumn{3}{|c|}{$3 \mathrm{~h}$} \\
\hline & \multicolumn{2}{|c|}{ No. of tags } & \multirow{2}{*}{$\begin{array}{l}\text { Fold } \\
\text { change }\end{array}$} & \multicolumn{2}{|c|}{ No. of tags } & \multirow{2}{*}{$\begin{array}{l}\text { Fold } \\
\text { change }\end{array}$} \\
\hline & Sham-TO & TO & & Sham-TO & TO & \\
\hline Aldolase 1, A isoform (BC043026) & 59 & 225 & $3.8 *$ & 112 & 256 & 2.3 \\
\hline$\alpha-S M A($ actin, $\alpha$, cardiac) (AK014303) & 45 & 294 & $6.5^{*}$ & 152 & 484 & $3.2 *$ \\
\hline Enolase 3, beta muscle (BC013460) & 5 & 79 & $15.8^{*}$ & 42 & 87 & 2.1 \\
\hline Glyceraldehyde-3-phosphate dehydrogenase (AK081405) & 60 & 190 & $3.2 *$ & 108 & 287 & $2.7 *$ \\
\hline Myosin heavy polypeptide 4, skeletal muscle (AJ278733) & 17 & 288 & $16.9 *$ & 149 & 480 & $3.2 *$ \\
\hline Myosin light chain, alkali, fast skeletal muscle (AK003182) & 7 & 88 & $12.6^{*}$ & 127 & 439 & $3.5^{*}$ \\
\hline $\begin{array}{l}\text { Myosin light chain, phosphorylatable, fast skeletal muscle } \\
\text { (AK010483) }\end{array}$ & 17 & 441 & $25.9 *$ & 183 & 676 & $3.7 *$ \\
\hline Phosphofructokinase, muscle (BC005526) & 1 & 37 & $37.0^{*}$ & 17 & 54 & 3.2 \\
\hline Phosphoglycerate mutase 2 (BC010750) & 2 & 58 & $29.0^{*}$ & 32 & 142 & $4.4^{*}$ \\
\hline Pyruvate kinase, muscle (BC016619) & 15 & 54 & $3.6^{*}$ & 34 & 63 & 1.9 \\
\hline Troponin C, fast skeletal (BC024390) & 15 & 293 & $19.5^{*}$ & 105 & 365 & $3.5^{*}$ \\
\hline Troponin I, fast skeletal 2 (BC028515) & 12 & 328 & $27.3^{*}$ & 161 & 564 & $3.5^{*}$ \\
\hline Troponin T3, fast skeletal (BC003747) & 2 & 114 & $57.0^{*}$ & 104 & 330 & $3.2 *$ \\
\hline Troponin T3, fast skeletal (L49467) & 14 & 197 & $14.1^{*}$ & 67 & 210 & $3.1 *$ \\
\hline
\end{tabular}

\footnotetext{
* Significant difference between timely corresponding sham-TO and TO groups.
} 
sham-TO lungs at $3 \mathrm{~h}$ than in sham-TO lungs at $1 \mathrm{~h}$. This observation raises two possibilities: 1) TO accelerates the decrease in the expression of defense-related proteins that normally takes place during lung development and 2) TO exacerbates the decrease in the expression of defense-related proteins in consequence of the surgery itself. It is also noteworthy to emphasize the fact that, among the 10 different tags corresponding with genes of this class, seven are related to homeostasis, whereas only three are associated with immune response. Homeostasis is a normal process in cells, particularly those subject to major stimulus, as is the case with lung expansion resulting from TO. Thus, this result does not necessarily suggest a tissue injury. Moreover, it has been demonstrated that some cell defense-related proteins have the potential, in addition to their protective role, to interact with other signaling networks and influence the outcome of complex developmental programs, such as limb (26) and organ regeneration (27).

During lung maturation, interstitial fibroblasts largely differentiate along either an adipogenic (lipofibroblasts) or a myogenic (myofibroblasts) phenotypic pathway (28). Myogenic and lipogenic phenotypes refer to cells presenting muscle-specific and adipose-specific gene and metabolism characteristics, respectively. It has been proposed that balance among different fibroblastic subpopulations is centrally important in signaling communication between the mesenchyme and epithelium. This signaling is known to be a crucial determinant of lung maturation (10). In utero, lipofibroblasts predominate within the perinatal lung where they participate in extracellular matrix structural proteins synthesis and are accessory cells of type II pneumocytes in surfactant synthesis (10). After birth, however, the proportion of lipofibroblasts decreases rapidly (10). In addition, it has been suggested that alveolar overdistention causes a decrease in the lipofibroblast number (29). Thus, the metabolic switch from lipid production and accumulation to glycolysis observed here could be the reflection of an increase in the myofibroblast/lipofibroblast ratio. Our histochemical data suggest that 1 and $3 \mathrm{~h}$ of TO has no measurable effect on lipid content in mesenchymal cells. However, $24 \mathrm{~h}$ of TO reduces the abundance of lipid phenotype compared with controls. This delay between transcriptional down-regulation and lipid phenotype diminution likely represents the time for the integration of new natural turnover of proteins and its consequence on actual presence of lipids. Moreover, TO cases at both 1- and 3-h time points possess significantly more mesenchymal $\alpha$-SMA-containing cells than correspondingly timed control cases. Furthermore, SAGE data reveal a pronounced up-regulation by TO of myogenic transcripts presumably associated with myofibroblasts (e.g. $\alpha$-SMA, myosin light chain, myosin heavy polypeptide 4 , troponins C, I, and T3 and others) (Table 2). However, the TO-driven increase in myofibroblastic phenotype, along with a relative decrease in lipofibroblastic phenotype in lung mesenchyme, has to be confirmed by more direct functional studies.

This study provides a novel comprehensive overview of cellular processes that are rapidly modulated by pulmonary intraluminal stretch increase resulting from TO. As outlined in

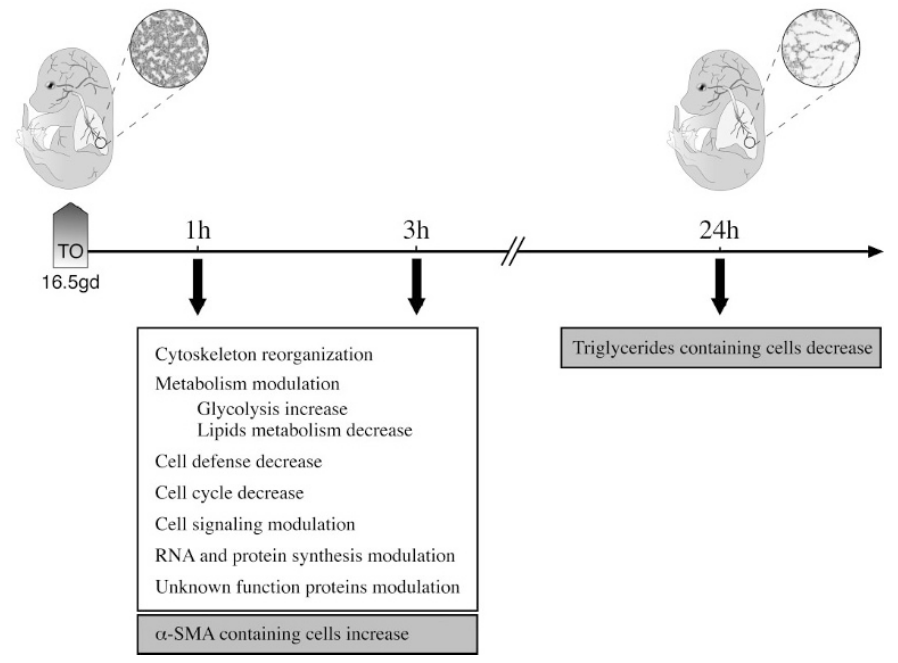

Figure 4. Summary and contextualization of cellular processes rapidly modulated by TO. TO was executed in mice at $16.5 \mathrm{~d}$ of gestation, when the fetal lung presents a limited size and immature morphology characterized by a poor surface exchange area. SAGE data (white box) delineated rapid transcriptional modulation of genes corresponding with various cellular processes that occur at 1 and $3 \mathrm{~h}$ after TO. In addition, immuno- and histochemical data (gray boxes) reveal an increase in $\alpha$-SMA-containing cells at 1 and $3 \mathrm{~h}$ as well as a relative decrease in triglyceride-containing cells in response to $24 \mathrm{~h}$ of TO, along with an observed increase in lung size and structural maturation (8).

Figure 4, we identified cellular processes that are modulated by $\mathrm{TO}$ before the previously reported increase in lung size and structural maturation that is observable $24 \mathrm{~h}$ after surgery (8). Overall, our data delineated cellular processes rapidly modulated by TO at a transcriptional level and also revealed an increase in $\alpha$-SMA-containing cells at 1 and $3 \mathrm{~h}$ as well as a relative decrease in triglyceride-containing cells at $24 \mathrm{~h}$. This study suggests that continuous stretching in a developing lung leads directly to rapid and highly specific phenotypic modifications in a significant proportion of pulmonary cells. Based on our data, we conclude that the intraluminal stretch increase during the prealveolar stage of lung development induces a critical transition of pulmonary cell phenotype in which there is an increase in $\alpha$-SMA-containing cells along with a relative decrease in lipid-containing cells. This investigation offers a comprehensive overview of cellular processes rapidly modulated by the pulmonary intraluminal stretch increase, providing a basis for further mechanistic analysis of TO response and potential novel avenues to understand the physiopathology of abnormal lung development associated with congenital diaphragmatic hernia, chronic loss of amniotic fluid, or, more frequently, extreme prematurity.

Acknowledgments. The authors thank Drs. Marc-André Dugas, Mélanie Kavanagh, and Pierre Savard for helpful discussions as well as Drs. Yves Tremblay and Pierre Provost for critical reading of the manuscript.

\section{REFERENCES}

1. Harding R, Hooper SB 1996 Regulation of lung expansion and lung growth before birth. J Appl Physiol 81:209-224

2. Patrick J, Campbell K, Carmichael L, Natale R, Richardson B 1980 Patterns of human fetal breathing during the last 10 weeks of pregnancy. Obstet Gynecol 56:24-30 
3. Hooper SB, Harding R 1995 Fetal lung liquid: a major determinant of the growth and functional development of the fetal lung. Clin Exp Pharmacol Physiol 22:235-247

4. Yoshimura S, Masuzaki H, Miura K, Hayashi H, Gotoh H, Ishimaru T 1997 The effects of oligohydramnios and cervical cord transection on lung growth in experimental pulmonary hypoplasia in rabbits. Am J Obstet Gynecol 177:72-77

5. Harding R, Bocking AD, Sigger JN 1986 Upper airway resistances in fetal sheep: the influence of breathing activity. J Appl Physiol 60:160-165

6. Miller AA, Hooper SB, Harding R 1993 Role of fetal breathing movements in control of fetal lung distension. J Appl Physiol 75:2711-2717

7. Hashim E, Laberge JM, Chen MF, Quillen EW Jr 1995 Reversible tracheal obstruction in the fetal sheep: effects on tracheal fluid pressure and lung growth. J Pediatr Surg 30:1172-1177

8. Maltais F, Seaborn T, Guay S, Piedboeuf B 2003 In vivo tracheal occlusion in fetal mice induces rapid lung development without affecting surfactant protein $\mathrm{C}$ expression. Am J Physiol Lung Cell Mol Physiol 284:L622-L632

9. Seaborn T, Khan PA, Cloutier M, Maltais F, Piedboeuf B 2007 Short-term response to tracheal occlusion during perinatal lung development in mice. Exp Lung Res $33: 441-457$

10. McGowan SE, Torday JS 1997 The pulmonary lipofibroblast (lipid interstitial cell) and its contributions to alveolar development. Annu Rev Physiol 59:43-62

11. St-Amand J, Okamura K, Matsumoto K, Shimizu S, Sogawa Y 2001 Characterization of control and immobilized skeletal muscle: an overview from genetic engineering. FASEB J 15:684-692

12. Chen H, Jackson S, Doro M, McGowan S 1998 Perinatal expression of genes that may participate in lipid metabolism by lipid-laden lung fibroblasts. J Lipid Res 39:2483-2492

13. Maniscalco WM, Watkins RH, Pryhuber GS, Bhatt A, Shea C, Huyck H 2002 Angiogenic factors and alveolar vasculature: development and alterations by injury in very premature baboons. Am J Physiol Lung Cell Mol Physiol 282:L811-L823

14. Laplante AF, Germain L, Auger FA, Moulin V 2001 Mechanisms of woun reepithelialization: hints from a tissue-engineered reconstructed skin to longstanding questions. FASEB J 15:2377-2389

15. De Paepe ME, Johnson BD, Papadakis K, Luks FI 1999 Lung growth response after tracheal occlusion in fetal rabbits is gestational age-dependent. Am J Respir Cell Mol Biol 21:65-76

16. Bolender RP, Hyde DM, Dehoff RT 1993 Lung morphometry: a new generation of tools and experiments for organ, tissue, cell, and molecular biology. Am J Physiol 265:L521-L548

17. Kim N, Vu TH 2006 Parabronchial smooth muscle cells and alveolar myofibroblasts in lung development. Birth Defects Res C Embryo Today 78:80-89
18. Velculescu VE, Zhang L, Vogelstein B, Kinzler KW 1995 Serial analysis of gene expression. Science 270:484-487

19. Meissner N, Radke J, Hedges JF, White M, Behnke M, Bertolino S, Abrahamsen M Jutila MA 2003 Serial analysis of gene expression in circulating gamma delta T cell subsets defines distinct immunoregulatory phenotypes and unexpected gene expression profiles. J Immunol 170:356-364

20. Gnatenko DV, Dunn JJ, McCorkle SR, Weissmann D, Perrotta PL, Bahou WF 2003 Transcript profiling of human platelets using microarray and serial analysis of gene expression. Blood 101:2285-2293

21. Dinel S, Bolduc C, Belleau P, Boivin A, Yoshioka M, Calvo E, Piedboeuf B, Snyder EE, Labrie F, St-Amand J 2005 Reproducibility, bioinformatic analysis and power of the SAGE method to evaluate changes in transcriptome. Nucleic Acids Res 33:e26

22. Trendelenburg G, Prass K, Priller J, Kapinya K, Polley A, Muselmann C, Ruscher K, Kannbley U, Schmitt AO, Castell S, Wiegand F, Meisel A, Rosenthal A, Dirnagl U 2002 Serial analysis of gene expression identifies metallothionein-II as major neuroprotective gene in mouse focal cerebral ischemia. J Neurosci 22:5879-5888

23. Muratore CS, Nguyen HT, Ziegler MM, Wilson JM 2000 Stretch-induced upregulation of VEGF gene expression in murine pulmonary culture: a role for angiogenesis in lung development. J Pediatr Surg 35:906-913

24. Hallauer PL, Hastings KE 2002 Coregulation of fast contractile protein transgene and glycolytic enzyme expression in mouse skeletal muscle. Am J Physiol Cel Physiol 282:C113-C124

25. Walker AM, Ritchie BC, Adamson TM, Maloney JE 1988 Effect of changing lung liquid volume on the pulmonary circulation of fetal lambs. J Appl Physiol 64:61-67

26. Del Rio-Tsonis K, Tsonis PA, Zarkadis IK, Tsagas AG, Lambris JD 1998 Expression of the third component of complement, $\mathrm{C} 3$, in regenerating limb blastema cells of urodeles. J Immunol 161:6819-6824

27. Mastellos D, Papadimitriou JC, Franchini S, Tsonis PA, Lambris JD 2001 A novel role of complement: mice deficient in the fifth component of complement (C5) exhibit impaired liver regeneration. J Immunol 166:2479-2486

28. Vaccaro C, Brody JS 1978 Ultrastructure of developing alveoli. I. The role of the interstitial fibroblast. Anat Rec 192:467-479

29. Torday JS, Torres E, Rehan VK 2003 The role of fibroblast transdifferentiation in lung epithelial cell proliferation, differentiation, and repair in vitro. Pediatr Pathol Mol Med 22:189-207 